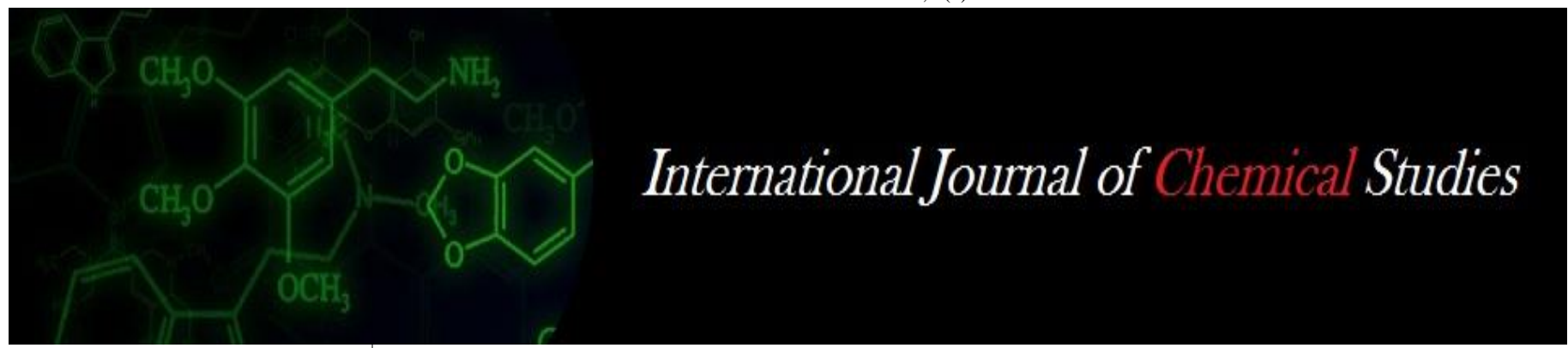

P-ISSN: 2349-8528

E-ISSN: 2321-4902

www.chemijournal.com

IJCS 2020; 8(5): 478-480

(C) 2020 IJCS

Received: 13-06-2020

Accepted: 15-08-2020

Luna Barooah

Assistant Professor, College of

Horticulture, Assam Agricultural

University, Jorhat, Assam, India

\section{Antioxidant property and phenol content of some medicinal plants from Sonitpur district of Assam}

\section{Luna Barooah}

DOI: https://doi.org/10.22271/chemi.2020.v8.i5g.10343

\begin{abstract}
The present experiment was conducted in the Department of Molecular Biology and Biotechnology, Tezpur University during 2007-08. In this experiment, nine indigenous medicinal plants are analysed for their total phenolic contents and antioxidant activity viz. Amaranthus spinosus, Centella asiatica, Chenopodium album, Houttuynia cordata, Moringa oleifera, Paederia foetida and Polygonum chinense. The antioxidant activity of methanol extracts of the plants were determined by Spectrophotometric method using the stable free radical DPPH (2, 2-diphenyl-1-picrylhydrazyl). The results revealed that Moringa oleifera leaves recorded highest total phenolic content and exhibited highest percent inhibition of free radical followed by Houttuynia cordata. While, the lowest total phenolic content and lowest percent inhibition of free radical was recorded in Chenopodium album.
\end{abstract}

Keywords: Phenol content, medicinal plants, Moringa oleifera

\section{Introduction}

There is an increasing interest in food industry and preventive health care for the development and evaluation of natural antioxidants from plants. Among the various medicinal plants, some are of particular interest because they may be used for the production of raw materials or preparations containing phytochemicals with significant antioxidant properties and health benefits (Exarchou et al., 2002) ${ }^{[6]}$. Plant derived antioxidants and phenolics have potential health benefits because of which they are very important for pharmaceutical industries. Among the potential uses of antioxidantsplays an important role in prevention of diseases related to oxidative stress in humans and also prevents oxidative reactions in food, pharmaceuticals, cosmetic products (Moure et al., 2001) ${ }^{[4]}$. Epidermological studies have shown that consumption of plant foods containing antioxidant is beneficial to health because it downregulates many degenerative processes and can effectively lower the incidence of cancer and cardiovascular diseases (Arabshahi and Urooj. 2007) ${ }^{[2]}$. Is mainly due to Phenolic components such as flavanoids, phenolic acids and phenolic diterpenes mainly exerts antioxidative effects (Shahidi, Janitha \& Wanasundara, 1992) ${ }^{[8]}$. The antioxidant activity of phenolic compounds is mainly due their redox properties, which can play an important role in absorbing and neutralizing free radicals, quenching single and triplet oxygen or decomposing peroxides (Osawa, 1994) ${ }^{[7]}$. Thus, it is important to increase the antioxidant intake in the diet and search for natural antioxidant in plants which can be used as food additives. In this study some of the indigenous medicinal plants are selected which are mostly used by people of Assam. The purpose of the study was to evaluate some of the indigenous medicinal plants as new potential sources of natural antioxidant and phenolic compounds.

\section{Materials and Methods}

Plant collection and extraction: The plant samples were collected from different places of Sonitpur district. The plant species included in the study are Amaranthus spinosus, Centella asiatica, Chenopodium album, Houttuynia cordata, Moringa oleifera, Paederia foetida and Polygonum chinense which have long been used in folk medicine of North East India due to their potential health promoting and pharmacological attributes. The plant materials were washed thoroughly with distilled water and shade-dried at room temperature. The dried leaves were ground well, powdered (about 12.5g) and extracted with methanol (125 ml) in shaking water bath at $50{ }^{\circ} \mathrm{C}$ for 3-5 times.
Corresponding Author:

Luna Barooah

Assistant Professor, College of

Horticulture, Assam Agricultural

University, Jorhat, Assam, India 
The extracts were filtered and concentrated with speed vacuum concentrator (Maxi Dry Plus). The dried extracts were stored at $4{ }^{\circ} \mathrm{C}$.

Total phenols: The total polyphenol content was determined by the Folin-Ciocalteau phenol reagent (Hagerman et al., 2000). $0.1 \mathrm{~mL}$ of diluted extracts was transferred to test tubes and their volumes made up to $0.5 \mathrm{~mL}$ with distilled water. After addition of $0.25 \mathrm{~mL}$ Folin-Ciocalteau reagent and $1.25 \mathrm{~mL} 20 \%$ aqueous sodium carbonate solution, tubes are vortexed and absorbance was recorded at $725 \mathrm{~nm}$ after $40 \mathrm{~min}$ against a blank solution containing $0.1 \mathrm{~mL}$ of the solvent instead of the tested sample. The total phenolic content was expressed as Gallic Acid Equivalents (GAE) in milligrams per gram of dry matter of sample, using a standard curve generated with different concentrations of Gallic acid.

DPPH Radical scavenging assay: The stable 1, 1-diphenyl2-picryl hydrazyl radical (DPPH) was used for determination of free radical-scavenging activity of the extracts (Koleva et al., 2002). Different concentrations of each herbal extracts were added, at an equal volume to methanolic solution of DPPH (100 microliter). After $15 \mathrm{~min}$ at room temperature, the absorbance was recorded t $517 \mathrm{~nm}$. The experiment was repeated for three times. BTH (Butylated Hydroxy Tolune) was used as standard controls. $\mathrm{IC}_{50}$ values represent the concentration of sample required to scavenge $50 \%$ of DPPH free radicals.
(Absorbance of control-Absorbance of sample)

$\%$ inhibition $=$

$$
\text { Absorbance of control }
$$

Statistical analysis: The data is stated as mean value $\pm \mathrm{SD}$ value. One-way ANOVA procedure was used to perform the analysis of variance. Minitab software was used to calculate the significant differences $(p<0.05)$ between mean values.

\section{Results \\ Antioxidant activity}

The proton free radical scavenging action is known to be one of the various mechanisms for measuring antioxidant activity. DPPH possess a proton free radical which shows a maximum absorption at $517 \mathrm{~nm}$. This method determines to scavenge stable radical of DPPH by antioxidants. Table1 shows the percent inhibition of DPPH at different concentration of the leaf extracts of the plants. From the table it is clear that percent inhibition increases with the increase in concentration of the extracts. Moringa oleifera recorded the highest percent inhibition of DPPH followed by Houttuynia cordata and polygonum chinense. Moringa oleifera is a rich source of ascorbic acid and flavonoids which also have the antioxidant activity (Anwar et al. 2005) ${ }^{[1]}$. P. foetida has higher levels of phenolic compounds than does $S$. aqueum, which may have contributed to its high antioxidant activity (Afroz et al., 2006) [3].

Table 1: Phenol content and antioxidant activity of plant species

\begin{tabular}{|c|c|c|c|c|c|}
\hline \multirow{2}{*}{$\begin{array}{c}\text { Leaf Extracts of } \\
\text { Plant species }\end{array}$} & \multirow{2}{*}{$\begin{array}{c}\text { Total phenol content } \\
(\mathbf{m g} / \mathbf{g} \text { of gallic acid } \\
\text { equivalents) }\end{array}$} & \multicolumn{4}{|c|}{ \% inhibition of DPPH or free radical scavenging effect at different concentrations } \\
\cline { 3 - 6 } & & $\mathbf{0 . 0 2 5}$ & $\mathbf{0 . 0 5 0}$ & $\mathbf{0 . 1 0}$ & $\mathbf{0 . 2 0}$ \\
\cline { 3 - 6 } & $46.78 \pm 0.02$ & $19.6 \pm 0.21$ & $22.4 \pm 0.23$ & $38.8 \pm 0.18$ & $46.6 \pm 0.22$ \\
\hline Amaranthus spinosus & $49.88 \pm 0.03$ & $32.5 \pm 0.15$ & $41.9 \pm 0.19$ & $46.1 \pm 0.12$ & $53.3 \pm 0.16$ \\
\hline Centella asiatica & $11.65 \pm 0.02$ & $21.7 \pm 0.21$ & $33.3 \pm 0.10$ & $42.5 \pm 0.14$ & $50.8 \pm 0.12$ \\
\hline Chenopodium album & $92.25 \pm 0.01$ & $20.5 \pm 0.16$ & $27.4 \pm 0.13$ & $74.4 \pm 0.12$ & $60.7 \pm 0.11$ \\
\hline Houttuynia cordata & $97.85 \pm 0.02$ & $56.2 \pm 0.12$ & $69.7 \pm 0.16$ & $52.2 \pm 0.11$ & $84.1 \pm 0.14$ \\
\hline Moringa oleifera & $21.34 \pm 0.03$ & $37.3 \pm 0.22$ & $43.6 \pm 0.25$ & $70.0 \pm 0.13$ & $48.1 \pm 0.10$ \\
\hline Leucas aspera & $35.34 \pm 0.02$ & $53.6 \pm 0.15$ & $62.3 \pm 0.20$ & $41.1 \pm 0.11$ & $52.4 \pm 0.11$ \\
\hline Paederia foetida & $15.76 \pm 0.01$ & $22.2 \pm 0.17$ & $30.4 \pm 0.14$ & $97.6 \pm 0.14$ & $97.9 \pm 0.15$ \\
\hline Polygonum chinense & - & $96.5 \pm 0.09$ & $97.2 \pm 0.12$ & & \\
\hline BHT (Control) & & & &
\end{tabular}

Values are the mean \pm standard deviation of triplicate

\section{Total phenol content}

The concentration of total phenoic compounds in all plant extracts was expressed as milligrams of gallic acid equivalents (GAE) per mg dry weight of plant. The amount of total phenolics varied in different plant species and ranged from $\mathrm{mg} \mathrm{GAE} / \mathrm{g}$ of dry material. The highest total phenolic content was detected in Moringa oleifera followed by Houttuynia cordata and lowest was recorded in Chenopodium album.

\section{Conclusion}

The findings of the experiment support that all of the extracts in this research exhibited different extent of antioxidant activity. A strong correlation between antioxidant activities and total phenol content indicated that phenolic compounds were a major contributor of antioxidant activity of these plants. M. olefera showed highest inhibition of free radical followed by Houttuynia cordata. This may be related to the higher amount of total phenol content in this plant extract. $M$. oleifera is known to have various medicinal benefits and these have been attributed to its phytochemicals such as phenolic compounds (Verma et al., 2009 \& Vongsak et al., 2013) ${ }^{[5,9]}$. From the experiment, it can be concluded that some of the plants are promising source of antioxidants and can be used a good source of natural antioxidants. Further, research need to be carried out on the isolation and identification of antioxidant compounds in the plant which may have different pharmaceutical uses.

\section{References}

1. Anwar F, Ashraf M, Bhanger MI. Interprovenance variation in the composition of Moringa oleifera oilseed from Pakistan. Journal of the American Oil Chemists Society. 2005; 82:45-51.

2. Arabshahi-Delouee S, Urooj A. Antioxidant properties of various solvent extracts of mulberry (Morus indica L.) leaves. Food Chem. 2007; 102:1233-1240.

3. Afroz S, Alamgir M, Khan MTH, Jabbar S, Nahar N, Choudhuri MSK. Antidiarrhoeal activity of the ethanol extracts of Paederia foetida Linn. (Rubiaceae) J Ethnopharm. 2006; 105:125-130. 
4. Moure JM, Cruz, Franco D. Natural antioxidants from residual sources, Food Chemistry. 2001; 72(2):145-171.

5. Verma R, Vijayakumar M, Mathela CS, Rao CV. In vitro and in vivo antioxidant properties of different fractions of Moringa oleifera leaves, Food and Chemical Toxicology. 2009; 47(9):2196-2201.

6. Exarchou V, Nenadis N, Tsimidou M, Gerothanassis IP, Troganis A, Boskou D. Antioxidant activities and phenolic composition of extracts from Greek oegano, Greek sage and summer savory. Journal of Agricultural \& Food Chemistry. 2002; 50(19):5294-5299.

7. Osawa T. Natural antioxidants for utilization in food and biological systems. Post harvest biochemistry of plant food materials in tropics. Japan Scientific Societies Press, Tokyo, Japan, 1994, 241-251.

8. Shahidi F, Janitha PK, Wanasundara PD. Phenolic antioxidants. Critical Reviews of Food Science \& Nutrition. 1992; 32(1):67-103.

9. Vongsak B, Sithisarn P, Mangmool S, Thongpraditchote S, Wongkrajang Y, Gritsanapan W. Maximizing total phenolics, total flavonoids contents and antioxidant activity of Moringa oleifera leaf extract by the appropriate extraction method, Industrial Crops and Products. 2013; 44:566-571. 